\title{
ARTIGOS
}

Submetido 17.12.2013. Aprovado 19.02.2015

Avaliado pelo processo de double blind review. Editor Científico: Felipe Zambaldi

DOI: http://dx.doi.org/10.1590/So034-759020150508

\section{MÉTODOS COLABORATIVOS NA GESTÃO DE CADEIAS DE SUPRIMENTOS: DESAFIOS DE IMPLEMENTAÇÃO}

\author{
Collaborative methods in supply chain management: implementation challenges \\ Métodos colaborativos en la gestión de cadenas de suministros: desafíos de \\ implementación
}

\begin{abstract}
RESUMO
O tema gestão colaborativa vem sendo abordado como forma de gerar vantagem competitiva. Iniciativas colaborativas em cadeias de suprimentos (CS) proporcionaram o surgimento de métodos e práticas que corroboram a ideia de que a competitividade não se dá mais entre empresas isoladas, e sim entre cadeias. Na literatura, além de métodos colaborativos para a gestão da cadeia de suprimentos (GCS), é possível encontrar iniciativas para projeto, configuração, otimização, entre outras. Porém, observa-se baixa adesão de métodos colaborativos para a GCS, assim como dificuldades de implementação. 0 objetivo do artigo é investigar os métodos colaborativos referentes à coordenação de CS e analisar suas características e dificuldades de implementação. Uma revisão sistemática apontou o Collaborative Planning, Forecasting and Replenishment (CPFR) como o método colaborativo mais abordado e, posteriormente, um estudo de caso identificou dificuldades na implementação que colaboraram com o que foi pesquisado na literatura.
\end{abstract}

PALAVRAS-CHAVE | Método colaborativo, cadeia de suprimentos, Collaborative Planning, Forecasting and Replenishment, gestão colaborativa, otimização.

\begin{abstract}
The collaborative management issue has been discussed as a mean to generate competitive advantage. Collaborative initiatives in supply chains (SC) enabled the emergence of methods and practices that support the idea that competitiveness does not occur between isolated companies any longer, but between chains. In literature, in addition to collaborative methods for supply chain management (SCM), it is possible to find initiatives for project, configuration, and optimization, among others. However, there is little adhesion of collaborative methods for SCM, as well as implementation difficulties. The purpose of the article is to investigate the collaborative methods concerning the coordination of SC and analyze their characteristics and implementation difficulties. A systematic review indicated the Collaborative Planning, Forecasting and Replenishment (CPFR) as the most discussed collaborative approach and, subsequently, a case study identified difficulties in the implementation that complemented what was researched in the literature.
\end{abstract}

KEYWORDS / Collaborative method, supply chain, Collaborative Planning, Forecasting and Replenishment, collaborative management, optimization.

LEONARDO DE CARVALHO GOMES

legomes.rs@gmail.com

Doutorando em Engenharia de

Produção pela Universidade Federal do Rio Grande do Sul, Programa de

Pós-Graduação em Engenharia de

Produção - Porto Alegre - RS, Brasil

\section{FRANCISCO JOSÉ KLIEMANN NETO}

kliemann@producao.ufrgs.br

Professor da Universidade Federal

do Rio Grande do Sul, Escola

de Engenharia, Departamento

de Engenharia de Produção e

Transportes - Porto Alegre - RS, Brasil

\section{RESUMEN}

El tema gestión colaborativa está siendo abordado como forma de generar ventaja competitiva. Iniciativas colaborativas en cadenas de suministros (CS) proporcionaron el surgimiento de métodos y práticas que corroboraron la idea de que la competitividad no ocurre más entre empresas aisladas, pero sí entre cadenas. En la literatura, allá de métodos colaborativos para la gestión de la cadena de suministros (CS) es posible encontrar iniciativas para proyecto, configuración, optimización, entre otras. Sin embargo, se observa baja adhesión de métodos colaborativos para la gestión de la cadena de suministros GCS, así como dificuldades de implementación. El objetivo de ese artículo es investigar los métodos colaborativos referentes a la coordinación de CS y analizar sus características y dificultades de implementación. Una revisión sistemática ha apuntado el Collaborative Planning, Forecasting and Replenishment (CPFR) como el método colaborativo más abordado y, posteriormente, un estudio de caso ha indentificado dificultades en la implementación que colaboran con lo que ha sido investigado en la literatura acerca del tema.

PALABRAS-CLAVE / Método colaborativo, cadena de suministros, Collaborative Planning, Forecasting and Replenishment, gestión colaborativa, optimización. 


\section{INTRODUÇÃO}

Em meados da década de 1990, perceberam-se movimentos no sentido de buscar um aumento de competitividade com iniciativas não só em empresas isoladamente mas também em arranjos interempresariais. Foi quando diversos conceitos foram apresentados, tais como filiére ou cadeias produtivas, cluster, supply chain (SC), condomínios industriais, entre outros. Com isso, percebe-se que a competitividade é, cada vez mais, buscada entre cadeias, e não entre empresas isoladamente (Kliemann \& Souza, 2004). Chan, Chong, e Zhou (2012) mostraram que as empresas operam atualmente em um complexo, imprevisivel e competitivo mercado global, e as organizações estão respondendo a esses desafios por meio da implementação de um gerenciamento colaborativo da cadeia de suprimentos (CS) para obterem vantagens competitivas. Assim, a busca pela competitividade está caracterizada pela colaboração entre empresas com o objetivo de atender as necessidades do consumidor de maneira ágil e econômica. Christopher (1998) aponta a integração como o núcleo de logística e gestão da cadeia de suprimentos (GCS) desde a década de 1980, sendo um tema relevante e fonte de melhoria para o desempenho operacional da CS.

O contexto CS foi significativamente explorado no sentido de medir, avaliar e gerenciar essas cadeias. Percebem-se, na literatura, métodos com características, aplicações, objetivos e limitações distintas. Além de métodos colaborativos para a gestão ou coordenação (também sinônimo de sincronização ou sistematização) da CS, é possível encontrar iniciativas para projeto, configuração, sincronização, otimização, entre outras. À medida que novos entrantes de competitividade e complexidade foram surgindo, os métodos colaborativos também foram contemplando esses fatores, e, com isso, aumentou a complexidade em suas implementações. Essa complexidade resulta em dificuldades na implementação dos métodos colaborativos e, consequentemente, numa baixa adesão por parte das empresas.

Sobre a aplicação de métodos colaborativos em CS, a primeira questão que surge é: quais são os métodos colaborativos atualmente disseminados e suas características? A resposta a essa pergunta foi a primeira proposição deste artigo, que se deu por meio de uma revisão sistemática em artigos de bases de dados internacionais. Em função da variedade de ferramentas, tipos de métodos e aplicações, a seleção dos artigos focou os métodos que permitem a coordenação da CS, e não iniciativas isoladas para tomada de decisão, projeto, configuração, seleção, entre outras.

A segunda questão sobre métodos colaborativos vem a ser sobre suas dificuldades de implementação. Apesar da disseminação do tema, ainda se constata baixa adesão aos métodos, embora não seja novidade a relevância nos dias atuais. Fabbe-Costes e Jahre (2007) suportam essa afirmação mostrando que apenas $1,5 \%$ dos artigos publicados em periódicos internacionais de 2000 a 2006, proeminentes sobre gestão e logística, aborda a GCS com o desempenho prático. Fisher (2007) comenta que nunca tanta tecnologia e capacidade intelectual foram dedicadas ao aprimoramento do desempenho das CS. Logo, a segunda proposição deste artigo busca responder à pergunta: quais são as potenciais causas das dificuldades e desafios na implementação de métodos colaborativos em CS? A resposta deu-se por meio de um estudo de caso, no qual se identificaram dificuldades e fatores na implementação do principal método colaborativo encontrado na revisão sistemática, os quais permitiram a comparação com a literatura pesquisada e posterior conclusões.

0 artigo está estruturado em duas partes. A primeira contém uma revisão sistemática sobre os principais métodos colaborativos estudados e aplicados atualmente. Também, consta uma revisão da literatura para melhor direcionamento da revisão sistemática. A segunda abrange um estudo de caso sobre a implementação não efetiva do principal método colaborativo encontrado, o Collaborative Planning, Forecasting and Replenishment (CPFR).

\section{MÉTODOS COLABORATIVOS E CADEIAS DE SUPRIMENTOS}

Este tópico contém uma breve revisão da literatura sobre arranjos empresariais, GCS, colaboração em CS e fatores de implementação (de êxito ou de dificuldade). A partir do entendimento desses conceitos preliminares, foi realizada a revisão sistemática e discussão dos resultados encontrados.

\section{Arranjos empresariais, colaboração e fatores de êxito ou de dificuldade}

Devido à grande competição entre empresas e mercados, o modo como elas vêm se organizando para gerar competitividade também tem se alterado, no sentido de formarem arranjos empresariais. Existem diversos tipos desses arranjos. Alguns autores classificam as unidades de análise das empresas em três principais: cadeia de valor (value chain) (Hines, 1993; Porter, 1990), cadeia de suprimentos (supply chain) (Ayers, 2001; Simchi-Levi, Kaminsky, \& Simchi-Levi, 2003) e cadeia produtiva (filiére) (Bandt, 1982). Como o conceito de cadeia produtiva e CS divergem entre alguns autores e convergem entre outros, neste artigo, será considerado CS todo arranjo produtivo que contenha um conjunto de relações comerciais e financeiras estabelecido entre os estados de transformação e um fluxo de troca entre fornecedores e clientes. 
A GCS, segundo Koh, Demirbag, Bayraktar, Tatoglu, e Zaim (2007), é definida pelo Conselho de Profissionais de Gestão da Cadeia de Suprimentos (CPGCS) como o planejamento e gerenciamento de todas as atividades envolvidas de aquisição e terceirização, conversão e todas as atividades de gestão de logística, bem como a coordenação e colaboração com parceiros. Para Chopra e Meindl (2001), a GCS inclui um conjunto de abordagens e práticas para integrar efetivamente fornecedores, fabricantes, distribuidores e clientes para melhorar o desempenho em longo prazo das empresas individuais e da CS, em um modelo de negócios coeso e de alto desempenho.

A colaboração dentro do processo de GCS geralmente ocorre quando há a troca de informações e tecnologias entre duas ou mais empresas, as quais dividem a responsabilidade do planejamento, gestão, execução e acompanhamento do desempenho. Para Cao e Zhang (2011), as empresas têm se esforçado para alcançar uma maior colaboração da CS e aproveitar os recursos e conhecimento de fornecedores e clientes.

Barratt e Oliveira (2001) apresentaram o conceito collaborative supply chain management (CSCM) como uma nova estratégia que evoluiu na década de 1990 e é motivada pelas abordagens anteriores como just in time (JIT), intercâmbio eletrônico de dados (EDI) e resposta rápida (QR). O CSCM permite compartilhar não só informações mas também o conhecimento, riscos e lucros, levando em conta todas as áreas funcionais que participam ao longo da CS. Mentzer (2001) corrobora e complementa que a colaboração na GCS pode ser definida como um meio pelo qual as empresas trabalham de maneira integrada com objetivos comuns. A partir desse conceito, tem-se percebido um direcionamento no sentido de coordenação da CS por meio do fluxo de informações, produtos e interação constante das empresas.

A colaboração apresenta divergências de conceitos na literatura. Segundo Cao e Zang (2011), a colaboração da cadeia de suprimentos (CCS) e a integração da cadeia de suprimentos (ICS), por vezes, têm sido usadas como sinônimos, pois ambas referem-se a um processo de acoplamento entre os parceiros. Para Frohlich e Westbrook (2001), a ICS tem sido definida como o grau de envolvimento com fornecedores e clientes. No entanto, a ICS significa o controle unificado de uma série de processos sucessivos anteriormente exercida de modo independente (Flynn, Huo, \& Zhao, 2010). Leuschner, Rogers e Charvet (2013) entendem ICS como o escopo e a força das ligações nos processos da CS entre empresas. Bellmunt e Torres (2013) mostraram ICS com mais ênfase no controle central, ou seja, na integração de processos regidos por contrato. Logo, os termos CCS (Stank, Keller, \& Daugherty, 2001) e Coordenação da CS (Carr, Kaynak, \& Muthusamy, 2008) são usados para descrever elementos da ICS, ou seja, a ICS é formada pela CCS e pela Coordenação da CS. Este artigo buscou métodos de coordenação caracterizados pela colaboração, ou seja, métodos de ICS.

Bellmunt e Torres (2013) explicam que a integração é um conceito multidimensional o qual abrange os diferentes níveis organizacionais da empresa: nível corporativo por meio de atitudes; nível estratégico por meio de padrões; operacional por meio de práticas. Os autores comentam também que, apesar de níveis corporativos e estratégicos poderem seguir um padrão, as práticas não o fazem. As práticas definidas por esses representam, neste artigo, os métodos colaborativos pesquisados e abordados.

Leuschner et al. (2013) realizaram uma metanálise e consolidaram dimensões para a ICS devido às divergências de foco: integração da informação; coordenação e compartilhamento de recursos; ligação organizacional e relacionamento; estratégica; operacional; tecnológica; práticas, atitudes; padrões; estratégico; sistemas; financeiro. No presente artigo, os métodos colaborativos enquadram-se na dimensão práticas. Porém, as dimensões coordenação e compartilhamento de recursos, operacional, tecnológica e sistemas também incluem os métodos colaborativos abordados neste artigo.

Flynn, Huo, e Zhao (2010) criticam a falta de foco na integração interna e apontam falhas em autores que se concentram na integração com clientes e fornecedores sem antes realizarem a integração dos processos internos das organizações. Os autores apontaram, em sua pesquisa, que a integração interna e a integração com clientes foram as atividades mais fortemente relacionadas com a melhoria do desempenho. No entanto, a integração com fornecedores é a iniciativa de integração mais comum observada nas empresas. Nakano (2009) mostra que atividade de previsão colaborativa interna tem um efeito positivo sobre a logística relativa ao desempenho da produção, mas a previsão colaborativa externa, não.

Nyaga, Whipple, e Lynch (2010) concordam que as relações colaborativas com parceiros melhoram eficiências, flexibilidade e sustentam vantagens competitivas, porém apontam que não estão claros quais fatores promovem o êxito. Para esses autores, a confiança e comprometimento são elementos interdependentes responsáveis pela melhoria do desempenho e satisfação das empresas dentro de um ambiente colaborativo como o compartilhamento de informações ou outro esforço conjunto. Para Mentzer (2001), alguns requisitos facilitam o processo de colaboração: liderança, expectativas claras, cooperação, confiança, divisão de benefícios ou tecnologia.

A confiança e o comprometimento são elementos que têm sido temas de várias pesquisas e possuem uma forte relação entre si e com o desempenho da CS. Nyaga et al. (2010) mostram que atividades de colaboração, tais como compartilhamento 
de informações, esforço conjunto de relacionamento e investimentos dedicados, levam à confiança e ao comprometimento, e estes, por sua vez, resultam em maior satisfação e desempenho. A pesquisa desses autores apontou que os compradores se concentram mais nos resultados do relacionamento, ou seja, os antecedentes da confiança são mais importantes, como o compartilhamento de informações, enquanto os fornecedores focam os objetivos alcançados com a colaboração, ou seja, os resultados da confiança, como a satisfação e desempenho. Kwon e Suh (2004) apontam que a falta de confiança cria altos custos de transação (verificação, inspeção e certificação dos seus parceiros comerciais), o que acarreta perda de produtividade, eficiência e eficácia, pilares das metas da CS.

Kwon e Suh (2004) criaram constructos para confirmar uma relação positiva e significativa entre o grau de comprometimento e o nível de confiança. 0 estudo mostra que a confiança da empresa em seu parceiro está altamente associada com os investimentos em ativos específicos de ambos os lados (positivamente) e com a incerteza comportamental (negativamente). 0 compartilhamento de informações reduz o nível de incerteza comportamental, que, por sua vez, melhora o grau de confiança. O nível de comprometimento está fortemente relacionado com o nível de confiança.

Cannon, Doney, Mullen, e Petersen (2010) alertam que a pesquisa e prática têm demonstrado que as relações entre comprador-fornecedor geram benefícios quando os parceiros exibem uma orientação em longo prazo. Os autores propõem que os efeitos relativos de confiança e desempenho na orientação de longo prazo são moderados pela cultura construída entre as organizações. Tanto Ganesan (1993) como Narayanan e Raman (2004) entendem que relacionamentos baseados em uma orientação de longo prazo permitem que empresas sacrifiquem ganhos em curto prazo em favor de benefícios para ambas em longo prazo. Três fatores têm um forte efeito na orientação em longo prazo: o desempenho de um fornecedor; a confiança do fornecedor no comprador; e a dependência do comprador em relação ao fornecedor (Doney \& Cannon, 1997; Monczka, Petersen, Handfield, \& Ragatz, 1998; Prahinski \& Benton, 2004).

As dificuldades na GCS iniciam-se desde a implementação de iniciativas até a sua gestão e amadurecimento. Andraski (2001) e Marks (2005) apontam os principais fatores de dificuldades nas implementações do CPFR: falta de conhecimento, treinamento, complexidade de implementação, falta de confiança, insegurança, dificuldades tecnológicas, processos internos não alinhados estrategicamente, barreiras culturais, barreiras econômicas, barreiras regulamentares, falta de liderança diretiva, filosofias de corporação diferentes. Porém, nota-se que esses fatores são comuns a qualquer iniciativa colaborativa e podem possuir relações de causa e efeito entre si. Kwon e Suh (2004) alertam que a falta de confiança impede a criação de atividades de valor agregado com parceiros e que ferramentas como vendor managed inventory (VMI), cross-docking (CD) e CFPR tornam-se ineficazes. Os autores criticaram tomadores de decisão que, muitas vezes, gastam tempo excessivo na análise do parceiro comercial em termos de credibilidade, confiabilidade e confiança, em vez de otimizarem suas operações.

Para Holweg e Pil (2008), a coordenação da CS depende da disponibilidade de informações rápidas, precisas e visíveis para todos, por isso a importância do uso e investimentos corretos em tecnologia de informação (TI) para não gerar uma dificuldade na ICS.

Outro fenômeno que pode prejudicar a ICS é o excessivo comportamento oportunista. Segundo Williamson (1985), o oportunismo é a forma mais forte de comportamento baseado no interesse próprio, uma característica humana que indica a procura egoísta e fundamentada na astúcia. 0 comportamento oportunista gera conflitos que podem levar a CS a se desfazer ou apresentar elos frágeis que comprometem o seu desempenho, mesmo em cadeias com elevado grau de coordenação e nas quais existe um alto nível de comprometimento entre os seus componentes.

Uma forma de atenuar esse problema e trazer certa previsibilidade às trocas econômicas são os contratos, formais ou informais, estabelecidos para criarem-se estruturas de governança (Begnis, Estivalete, \& Pedrozo, 2007). Baiman e Rajan (2002) apresentam a necessidade de um trade-off entre a eficiência produtiva e o oportunismo nas relações, pois, à medida que o comprador repassa informações e tecnologias ao fornecedor, resolve-se os problemas de ineficiência, mas, com o tempo, o custo sobre o uso oportunista do fornecedor é tão grande que o comprador opta por renunciar aos benefícios de eficiência oriunda da exploração de uma inovação dentro da CS.

Hill (1990) faz uma crítica aos teóricos dos custos de transação, afirmando que eles geralmente negligenciam os mecanismos de autoajustamento dos mercados ao considerar o risco de comportamentos oportunistas, pois o mercado tratará de eliminar aqueles atores que habitualmente se comportam de maneira oportunista.

A partir do conceito CSCM, direcionou-se a revisão sistemática do próximo tópico no sentido de buscar métodos colaborativos voltados para a coordenação da CS, e não métodos, iniciativas ou ferramentas para tomada de decisão, projeto, configuração, seleção de parceiros, otimização operacional, entre outros.

\section{Revisão sistemática}

Este tópico apresenta a revisão sistemática, os métodos encontrados e os apresenta conceitualmente. A seleção dos métodos foi focada naqueles que atuam na coordenação colaborativa da CS. 


\section{Pesquisa e resultados de busca}

A pesquisa foi restringida a artigos publicados nos últimos 10 anos, com foco nas áreas afins e nas principais bases de dados nacionais e internacionais: ScienceDirect (Elsevier), ISI Web of Knowledge, SciELO, Emerald. Inicialmente, a busca foi realizada com as palavras-chave "método" e "colaborativo" para selecionar artigos referentes a métodos colaborativos em CS. Como o resultado mostrou baixa incidência, apenas 12 artigos, realizou-se uma nova busca com as palavras-chave "colaborativo" e "supply chain”. Após a busca eletrônica, selecionaram-se manualmente aqueles que apresentaram um método colaborativo referente à coordenação da CS. A Tabela 1 mostra esse resultado.

\section{Tabela 1. Resultados da segunda busca dos artigos}

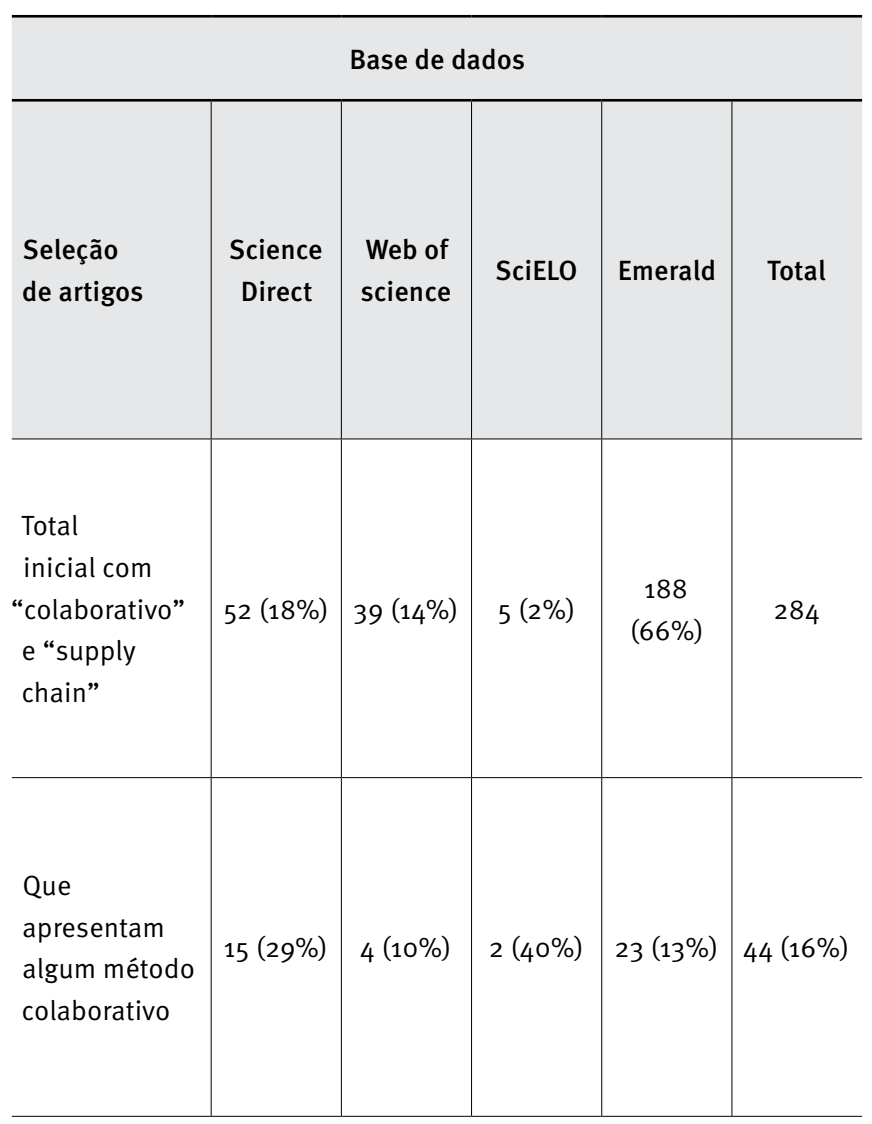

A Tabela 2 apresenta os métodos colaborativos encontrados nos artigos selecionados, alguns dos quais abordaram mais de um método. Os outros métodos encontrados na busca abordam diversos temas, tais como impacto de atividades colaborativas, panoramas de colaboração, ferramentas para análise de falhas em CS, estudos de caso de colaboração em diversos segmentos, análise de relações colaborativas, ferramentas Radio-Frequency IDentification (RFID), confiança nas relações colaborativas, entre outros.
Tabela 2. Métodos apresentados nos artigos selecionados

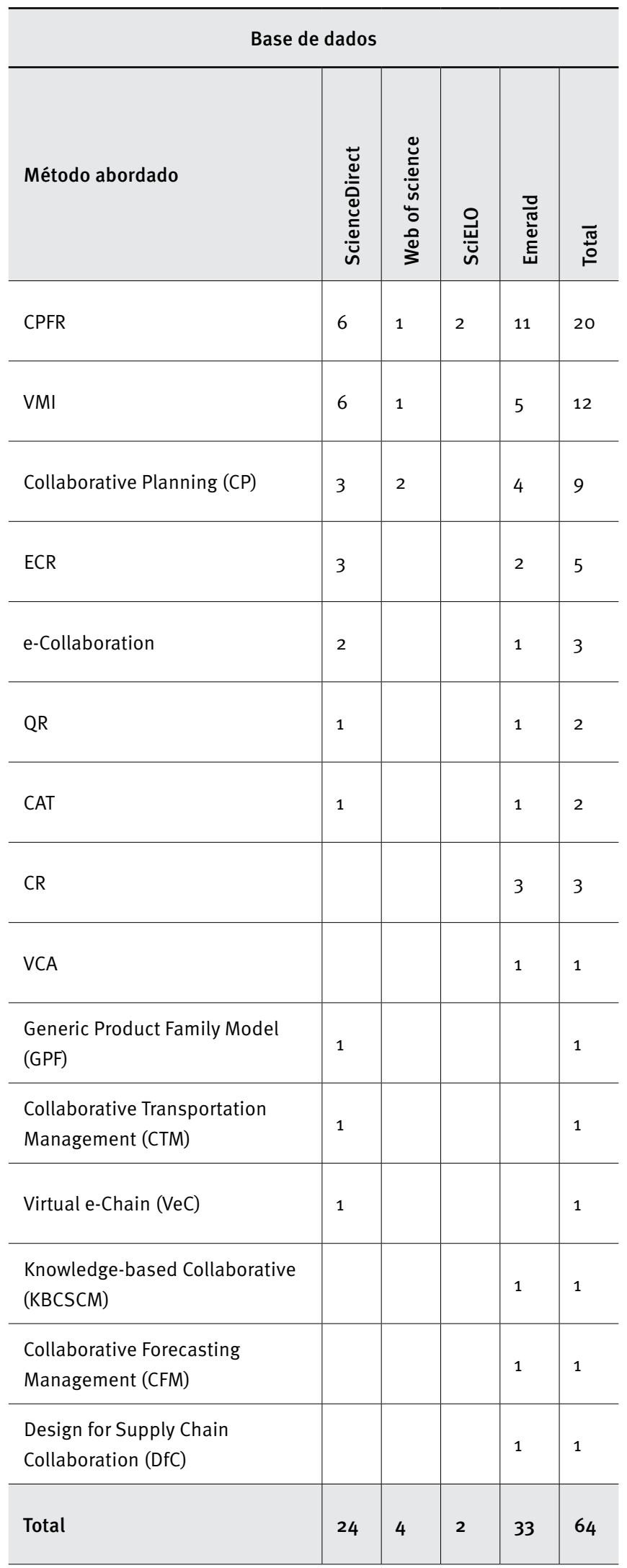


O Quadro 1 mostra uma análise crítica de todos os métodos colaborativos selecionados.

Quadro 1. Caracterização dos métodos colaborativos selecionados

(continua)

\begin{tabular}{|c|c|c|}
\hline Método Abordado & Autores & Escopo \\
\hline $\begin{array}{l}\text { Collaborative } \\
\text { Planning } \\
\text { Forecasting } \\
\text { Replenishment } \\
\text { (CPFR) }\end{array}$ & $\begin{array}{l}\text { Attaran e Attaran (2007) } \\
\text { Bailey e Francis (2008) } \\
\text { Cassivi (2006) } \\
\text { Chan e Zhang (2011) } \\
\text { Chang et al. (2007) } \\
\text { Choi e Suresh (2010) } \\
\text { Danese (2007) } \\
\text { Disney, Naim, e Potter (2004) } \\
\text { Du et al. (2009) } \\
\text { Fliedner (2003) } \\
\text { Pramatari (2007) } \\
\text { Ramanathan (2012) } \\
\text { Sari (2008) } \\
\text { Son e Chwen (2008) } \\
\text { Sparks e Beverly (2003) } \\
\text { Thron, Nagy, e Gábor (2007) } \\
\text { Thron, Nagy, e Wassan (2006) } \\
\text { Vivaldini, Pires, e Souza (2010) } \\
\text { Vivaldini, Souza, e Pires (2008) } \\
\text { Whipple e Russell (2007) } \\
\text { Yao et al. (2013) }\end{array}$ & $\begin{array}{l}\text { Planejamento colaborativo da demanda final e da reposição dos estoques entre os } \\
\text { participantes. Possui uma complexidade de implementação devido ao escopo da } \\
\text { metodologia. É o método colaborativo mais completo e proativo, configurando-se } \\
\text { como uma extensão das demais iniciativas como ECR, VMI e CR. }\end{array}$ \\
\hline $\begin{array}{l}\text { Vendor } \\
\text { Management } \\
\text { Inventory (VMI) }\end{array}$ & $\begin{array}{l}\text { Bailey e Francis (2008) } \\
\text { Barratt (2003) } \\
\text { Borade, Kannan, e Bansod } \\
\text { (2013) } \\
\text { Choi e Suresh (2010) } \\
\text { Danese (2007) } \\
\text { Disney, Naim, e Potter (2004) } \\
\text { Pramatari (2007) } \\
\text { Sari (2008) } \\
\text { Thron, Nagy, e Wassan (2006) } \\
\text { Vlist, Kuik, e Verheijen (2007) } \\
\text { Whipple e Russell (2007) } \\
\text { Yao, Evers, e Dresner (2007) }\end{array}$ & $\begin{array}{l}\text { O fornecedor realiza todo o gerenciamento dos estoques de seus produtos no } \\
\text { cliente. Atua de maneira localizada (entre duas empresas) em uma lógica reativa, } \\
\text { pois não considera a previsão de vendas. }\end{array}$ \\
\hline $\begin{array}{l}\text { Collaborative } \\
\text { Planning (CP) }\end{array}$ & $\begin{array}{l}\text { Barratt (2003) } \\
\text { Barratt (2004) } \\
\text { *Cassivi (2006) } \\
\text { Chiu e Lin (2004) } \\
{ }^{\star} \text { Cuenca et al. (2013) } \\
\text { Taghipour e Frayret (2013) } \\
\text { Vaart e Wijngaard (2007) }\end{array}$ & $\begin{array}{l}\text { Método não estruturado e focado na tomada de decisão sobre planos ao longo da } \\
\text { CS. Requer maturidade dos agentes para a melhor tomada de decisão. Não foca } \\
\text { necessariamente a previsão de demanda. Método baseado em algoritmos como o } \\
\text { ERP. }\end{array}$ \\
\hline $\begin{array}{l}\text { Efficiente } \\
\text { Consumer } \\
\text { Response (ECR) }\end{array}$ & $\begin{array}{l}\text { Bailey e Francis (2008) } \\
\text { ^Disney, Naim, e Potter (2004) } \\
\text { Sparks e Beverly (2003) } \\
\text { Whipple e Russell (2007) }\end{array}$ & $\begin{array}{l}\text { Método estruturado em quatro pilares: reposição, seleção, promoção e introdução } \\
\text { de novos produtos. Caracterizado como reativo à demanda. Possui como processo- } \\
\text { chave o gerenciamento por categorias e a reposição contínua de produtos. Objetivo } \\
\text { é buscar padrões comuns, eficiência em toda a CS e melhor serviço ao consumidor. }\end{array}$ \\
\hline
\end{tabular}


Quadro 1. Caracterização dos métodos colaborativos selecionados

(conclusão)

\begin{tabular}{|c|c|c|}
\hline Método Abordado & Autores & Escopo \\
\hline e-Collaboration & $\begin{array}{l}\text { Cassivi (2006) } \\
\text { Chan, Chong, e Zhou (2012) } \\
\text { Chong, Ooi, e Sohal (2009) }\end{array}$ & $\begin{array}{l}\text { Método não estruturado e dependente de ferramentas de colaboração. Permite a } \\
\text { integração dos processos de negócio e compartilhamento de informações entre } \\
\text { os membros da CS. Aproveita a internet para criar e manter uma comunidade de } \\
\text { negócios interativos de parceiros, fornecedores e clientes. }\end{array}$ \\
\hline $\begin{array}{l}\text { Quick Response } \\
\text { (QR) }\end{array}$ & $\begin{array}{l}\text { Choi e Sethi (2010) } \\
\text { Sparks e Wagner (2003) }\end{array}$ & $\begin{array}{l}\text { Iniciativa que surgiu na indústria de confecções com objetivo de integrar } \\
\text { fornecedores e varejistas na reposição de estoques. Característica reativa à } \\
\text { demanda e de simples implementação. }\end{array}$ \\
\hline $\begin{array}{l}\text { Collaborative } \\
\text { Agent Time (CAT) }\end{array}$ & *Carle, Martel, e Zufferey (2012) & $\begin{array}{l}\text { Método metaheurístico híbrido baseado no conceito de equipes de agentes } \\
\text { assíncronos (A-Teams). O método é voltado para solucionar problemas complexos } \\
\text { de projetos e operação de fornecedores, produção e distribuição. O CAT tem caráter } \\
\text { de solucionar problemas, e não de trabalhar na sincronização da CS. }\end{array}$ \\
\hline $\begin{array}{l}\text { Value chain } \\
\text { analysis (VCA) }\end{array}$ & Simons e Bourlakis (2008) & $\begin{array}{l}\text { Voltado para a otimização operacional. Método busca constantemente a } \\
\text { identificação dos gargalos e desperdícios que dificultam o funcionamento da CS. } \\
\text { Pouco voltado para a coordenação da CS. }\end{array}$ \\
\hline $\begin{array}{l}\text { Generic Product } \\
\text { Family Model } \\
\text { (GPF) }\end{array}$ & Jiao e Helander (2006) & $\begin{array}{l}\text { Focado na customização em massa com avaliação e geração de ordens de produtos } \\
\text { customizados. A coordenação da CS não contempla, neste método, fatores como } \\
\text { estratégias de reposição de estoques e previsão de demanda. }\end{array}$ \\
\hline $\begin{array}{l}\text { Collaborative } \\
\text { Transportation } \\
\text { Management } \\
\text { (CTM) }\end{array}$ & Chan e Zhang (2011) & $\begin{array}{l}\text { Focado na interação dos participantes da CS para agilizar entregas e reduzir custos } \\
\text { logísticos. Focado na logística e voltado mais para otimização do que coordenação } \\
\text { da CS. }\end{array}$ \\
\hline $\begin{array}{l}\text { Knowledge-based } \\
\text { Collaborative } \\
\text { (KBCSCM) }\end{array}$ & Udin, Khan, e Zairi (2006) & $\begin{array}{l}\text { Focado no aprendizado e conhecimento que busca resolver problemas rotineiros } \\
\text { nas CS. Método pouco estruturado e com o uso de ferramentas de aprendizagem. } \\
\text { Pouco focado na sistematização da CS. }\end{array}$ \\
\hline $\begin{array}{l}\text { Collaborative } \\
\text { Forecasting } \\
\text { Management } \\
\text { (CFM) }\end{array}$ & Rodriguez et al. (2008) & $\begin{array}{l}\text { Focado na demanda para a redução dos níveis de estoques. Pouco estruturado em } \\
\text { relação ao CPFR e não contempla a reposição de estoques. }\end{array}$ \\
\hline $\begin{array}{l}\text { Design for } \\
\text { Supply Chain } \\
\text { Collaboration } \\
\text { (DfC) }\end{array}$ & Simatupang e Sridharan (2008) & $\begin{array}{l}\text { Focado na construção de uma CS colaborativa. Não possui características de } \\
\text { coordenação da CS, pois esse tópico torna-se esporádico neste método. }\end{array}$ \\
\hline
\end{tabular}

*Encontrado nas bases de dados Emerald e ScienceDirect 


\section{Resultados e conclusões preliminares}

Uma característica comum entre todos os métodos encontrados é o compartilhamento de informações. Bailey e Francis (2008) apontam o compartilhamento de informações na CS como o fator-chave para a melhoria do desempenho, mas também são necessários fatores técnicos colaborativos para trabalhar e tomar decisões baseado nessas informações, e não simplesmente o compartilhamento de informações. Esses autores apresentam o método ECR como o método pioneiro a partir dos anos 1990 no Reino Unido, onde práticas colaborativas focavam a redução de inventários e a agilidade do fluxo de informações. Em práticas colaborativas, as informações são referentes a: confiança e abertura de informações; estratégia e visão compartilhada; alinhamento de indicadores; tomada de decisão e previsão de demanda; padronização de práticas e controles; incentivos; projetos; melhoria da CS. O principal método para esse tipo de colaboração tem sido o CPFR. Gomes (2015) mostra o CPFR como uma extensão de métodos como o ECR devido seu foco ser mais abrangente e pró-ativo em termos de previsão de demanda e tomada de decisões estratégicas conjuntas sobre as variações de demanda existentes. Isso pode ser verificado por sua incidência na pesquisa (Tabela 2) e por suas características de colaboração e coordenação de CS (Quadro 1).

Skjoett-Larsen, Thernøe, e Andresen (2003) referem-se ao CPFR como uma abordagem geral ou mecanismo para a coordenação dos processos entre os participantes da CS, que, mais especificamente, estabelece relações mais estreitas entre varejista e fornecedor, sincronização de previsões e processos de ressuprimentos, de modo a se tornar uma solução para o aumento da competitividade. Estes autores apontam o CPFR como uma abordagem mais holística, estruturada e totalmente colaborativa. Ireland (2005) aponta o CPFR como método colaborativo para promover atualmente a colaboração com o fornecedor. Ramanathan e Gunasekaran (2012) colocam o CPFR como a nova ferramenta para desenvolver a CCS. Sari (2008) aponta uma superioridade do CPFR sobre o VMI, mas atenta para os requisitos mais complexos do CPFR. Já o VMI é usado para reduzir as variações de estoque no fornecedor, pois o estoque do cliente é gerenciado pelo fornecedor. Ramanathan (2012) mostra o CPFR como método que incentiva todos os parceiros da CS a trocarem informações constantemente sobre suas previsões de demanda e, de maneira metódica, chegar a um consenso sobre planejamento.

O método CP pode ser definido como um processo de tomada de decisão conjunta para alinhar os planos com objetivos e alcançar certo grau de coordenação (Stadtler, 2009). Segundo Cuenca, Boza, Alemany, e Trienekens (2013), o CP é um processo complexo de coordenação por meio de mecanismos específicos, em que, ao longo do tempo, se cria a maturidade colaborativa.

O VMI, ECR, CP, CR e QR caracterizam-se como passos anteriores ao CPFR, enquanto os outros métodos selecionados possuem características específicas e pouco voltadas para a coordenação da CS.

O seguinte tópico apresenta o segundo objetivo do artigo: apontar dificuldades e desafios na implementação dos métodos colaborativos em CS, a partir um estudo de caso do CPFR.

\section{ESTUDO DE CASO}

A partir do segundo objetivo deste artigo, que busca as potenciais causas de dificuldades na implementação de métodos colaborativos em CS, a seleção do objeto do estudo de caso deu-se mediante dois critérios. 0 primeiro foi identificar alguma implementação do CPFR, devido ao seu enfoque na revisão sistemática anterior. 0 segundo foi a identificação de um estudo de caso do CPFR em que houvessem ocorrido dificuldades na implementação com o intuito de se analisarem as causas. Este tópico esquematiza o CPFR e, posteriormente, apresenta o estudo de caso em si.

\section{COLLABORATIVE PLANNING, FORECASTING AND REPLENISHMENT}

Em meados da década de 1990, o projeto piloto do CPFR ocorreu entre Walmart e Warner-Lambert. A partir disso, o modelo passou por atualizações em 2001, por meio do Voluntary Interindustry Commerce Stand (VICS), e, em 2004, o VICS desenvolveu uma revisão mais abrangente, visando integrar inovações e superar os problemas identificados no projeto original. Desde sua publicação, muitos autores contribuíram para a disseminação do CPFR, como Cederlund, Kohli, Sherer, e Yao (2007), que estudaram casos de implementação na Motorola, e Smith (2006), que analisou o caso da West Marine.

Sheffi (2002) indica o CPFR como a mais recente e mais completa forma de gestão colaborativa de negócios.

O CPFR concentra-se na elaboração conjunta de previsão de vendas e no planejamento de reposição dos itens envolvidos, levando em consideração as limitações existentes na CS, sejam elas do fornecedor industrial, do cliente varejista ou do distribuidor (Andraski, 2002).

O processo de funcionamento do CPFR, assim como seus elementos, é mostrado na Figura 1. 
Figura 1. Tarefas do fabricante e varejista nas fases do CPFR

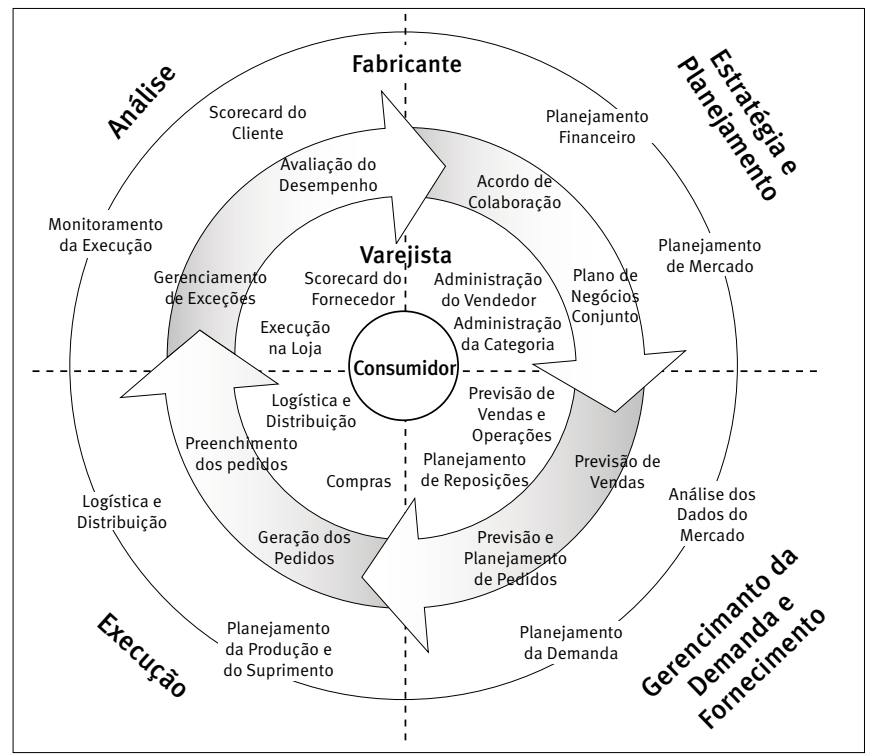

Fonte: VICS, 2004

No setor de varejo, um varejista em geral cumpre o papel do comprador, um fabricante cumpre o papel do vendedor e 0 consumidor é o cliente final. Em outros segmentos da indústria, tais como alta tecnologia, os participantes colaborativos podem ser diferentes. Por exemplo, um fabricante de equipamentos originais (Original Equipment Manufacturer - OEM), no papel do comprador, pode montar eletrônicos de fornecedores de componentes e, no papel de vendedor, pode entregar o produto para o cliente final (VICS, 2004).

O CPFR pode ser estendido para abranger mais de dois níveis de negociação entre parceiros, incluindo, por exemplo, fornecedores dos fabricantes.

Seifert (2002) apresentou o processo de implementação da VICS começando por um acordo inicial de colaboração e intenções, passando pela ciração de um plano de negócios conjunto para a obtenção dos objetivos; em seguida, cria-se a sistemática de previsão de vendas e tomada de decisão, em que ocorre a identificação e resolução das exceções à previsão de vendas. Após esse planejamento macro, entra a parte da previsão dos pedidos em si, com a identificação e resolução das exceções. Após essa etapa, vem a geração dos pedidos e retroalimentação do sistema, aprimorando-o. Em cada etapa, são definidos os participantes e a periodicidade das interações.

O CPFR possui certa complexidade na implementação, por envolver fatores técnicos e fatores não técnicos e, ainda, por ser um processo em que a implementação passa por vários ciclos de funcionamento até estabilizar o sistema. Andraski (2001) e Marks (2005) apontaram os principais fatores de dificuldades nas implantações do CPFR, citados no item Arranjos empresariais, colaboração e fatores de êxito ou de dificuldade deste artigo.

Fliedner (2003) acrescenta como dificuldade para a implementação do CPFR o medo de colisão, ou seja, as empresas nem chegam a tentar uma iniciativa colaborativa com medo de futuros conflitos estratégicos.

A confiança apontada por Andraski (2001) também é indicada como elemento de sucesso em diversos estudos (Danese, 2007; Min et al., 2005; Skjoett-Larsen et al., 2003).

Andraski (2003) atenta que o não alinhamento de metas estratégicas é um fator que tende a deixar as empresas em sua "zona de conforto", prejudicando qualquer ação colaborativa. Atenta também que devem ser expostos os ganhos financeiros para ambos os participantes do CPFR. Attaran e Attaran (2007) defendem os sistemas de informação abertos para facilitar o vínculo entre as empresas.

\section{ESTUDO DE CASO: COLLABORATIVE PLANNING, FORECASTING AND REPLENISHMENT}

O estudo de caso foi realizado por meio de uma abordagem qualitativa de acordo com Mattar (1997), que orienta para a identificação da presença ou ausência de algo, contribuindo para a elaboração de teorias sobre o fenômeno estudado. A abordagem qualitativa do estudo de caso foi conduzida por meio de entrevistas abertas com participantes selecionados. 0 estudo foi dividido em três etapas: cenário e identificação do objeto do estudo de caso; definição da amostra, planejamento e realização das entrevistas; e descrição, análise e interpretação dos resultados.

Na primeira etapa, parte de uma cadeia moveleira do estado do Rio Grande do Sul/Brasil foi adotada como objeto do estudo de caso. Esta escolha se deve ao fato que além de a implantação do CPFR não ter sido efetiva, a mesma gerou um aprendizado na empresa que liderou o processo de implementação e direcionou a empresa para a implementação de bases necessárias ao CPFR. A parte da cadeia estudada contempla a fabricação, embalamento e envio de adesivos para móveis. Essa parte da cadeia contém três empresas: fornecedora de latas para os adesivos, fabricante dos adesivos (proponente do CPFR) e distribuidora e detentora da marca do adesivo.

A empresa fornecedora de latas é uma metalúrgica que, desde seu início, em 2004, realiza a produção de embalagens metálicas. A empresa fornecedora de adesivos e proponente do CPFR atua nos segmentos calçadista, moveleiro, embalagem, transportes e construção civil. A empresa possui o perfil de uma fornecedora de commodities, ou seja, fornece produtos padroni- 
zados em alto volume para o mercado, mas com uma variedade de produtos em função dos segmentos em que atua. A empresa de adesivos e detentora da marca recebe as embalagens envasadas com os adesivos, realiza inspeções de qualidade por amostragem e distribui os produtos em grandes redes de lojas.

A implementação do CPFR foi liderada pela empresa fabricante de adesivos, pelo gerente de compras e logística. 0 tempo de planejamento do sistema levou aproximadamente um ano. Após esse período, o processo ficou a cargo do sucessor do gerente de compras e logística, que participou do processo de planejamento do CPFR e atuava na logística de suprimentos. A implementação do CPFR teve um curto período de execução, de seis meses, e não se consolidou.

A segunda etapa contemplou a definição da amostra, planejamento e realização das entrevistas. A amostra levou em consideração a maior extensão possível, ou seja, foram entrevistadas as pessoas-chave que lideraram e participaram da implementação. Foram elas:

Empresa fornecedora de latas (F):

F1) Gerente Geral, articulador da parceira;

F2) Gerente Comercial, que acompanhou o processo.

Empresa de adesivo e proponente do CPFR (P):

P1) Ex-Gerente de Logística e Compras, líder da implementação do CPFR;

$\mathrm{P} 2)$ Gerente de Logística e posterior líder da implementação do CPFR.

Empresa final de adesivos (C):

C1) Gerente de Compras, que participou do processo na época. 0 roteiro das entrevistas foi dividido em quatro etapas:

- Para quê? Objetiva investigar como veio a necessidade de ações colaborativas e como foi a origem da escolha na época pelo CPFR.

- Como foi? Objetiva investigar como foi o processo de implementação e quais as vantagens percebidas.

- Problema? Objetiva investigar o que houve de errado, quais foram as dificuldades e quais foram os fatores críticos no processo.

- Alternativas? Objetiva investigar como está a situação atualmente, como lidam com a questão de GCS e qual foi a alternativa técnica adotada.

As entrevistas foram realizadas de modo individual, com duração média de uma hora e gravadas para uma melhor descrição posterior dos dados.

A terceira etapa consistiu nas fases de descrição, análise e interpretação. Os dados das entrevistas foram descritos para, posteriormente, ocorrer a análise dos resultados, etapa que se caracteriza pela ordenação e agrupamento dos dados. A interpretação caracteriza-se pelo questionamento dos dados analisados e conclusões.

\section{Descrição e análise dos dados}

A primeira análise a partir da descrição das entrevistas foi sobre a necessidade da implementação de métodos colaborativos. Segundo o ex-Gerente de Logística, líder do projeto CPFR, essa necessidade ocorreu em 2003, quando as divergências entre as compras dos clientes e as previsões internas ocasionavam descontrole nos estoques. Segundo ele: "O índice de acerto era baseado nas informações de clientes e históricos de vendas e não gerava um bom resultado. Tínhamos um processo mais reativo do que colaborativo e precisávamos de algo que evitasse a falta ou estoque excessivo. O CPFR era o método mais avançado". A escolha do segmento moveleiro, segundo ele, deu-se por demonstrar uma melhor previsibilidade de demanda.

O Quadro 2 mostra as percepções dos entrevistados. Conforme mostrado anteriormente, as siglas equivalem aos entrevistados.

\section{Quadro 2. Percepções sobre necessidade de métodos colaborativos como o CPFR}

\begin{tabular}{|c|c|c|c|c|c|}
\hline & $\mathrm{F}_{1}$ & $F_{2}$ & $\mathrm{P}_{2}$ & $P_{1}$ & $C_{1}$ \\
\hline $\begin{array}{l}\text { Necessidade } \\
\text { de otimização }\end{array}$ & $x$ & $x$ & $x$ & $x$ & \\
\hline $\begin{array}{l}\text { Problema } \\
\text { específico }\end{array}$ & & & $X$ & $x$ & \\
\hline $\begin{array}{l}\text { Novos } \\
\text { negócios }\end{array}$ & $x$ & $x$ & $X$ & $x$ & $x$ \\
\hline
\end{tabular}

A empresa fornecedora de latas integrou-se no CPFR como uma oportunidade de melhoria e fidelização do cliente, enquanto a empresa final de adesivos, segundo o Gerente de Compras: "viu uma possibilidade de novo negócio, onde nos preocuparíamos apenas com a distribuição e não mais em envazar internamente". Porém, somente a fornecedora de adesivos proponente do CPFR entendeu que o estímulo à implementação também veio de um problema específico, que era a falta de assertividade das previsões de demanda. Essa condição é comum, uma vez que, enquanto fornecedores buscam redução de custos, clientes esperam receber produtos com a qualidade certa, no momento certo e com custo adequado.

A segunda análise foi sobre as percepções dos entrevistados quanto às vantagens dos métodos colaborativos como o CPFR. O Quadro 3 mostra as percepções dos entrevistados. 
Quadro 3. Percepções sobre as vantagens de métodos colaborativos como o CPFR

\begin{tabular}{|l|c|c|c|c|c|}
\cline { 2 - 6 } \multicolumn{1}{c|}{} & F1 & F2 & $\mathrm{P}_{1}$ & $\mathrm{P}_{2}$ & $\mathrm{C}_{1}$ \\
\hline $\begin{array}{l}\text { Enxergar e gerenciar a demanda do } \\
\text { cliente }\end{array}$ & $\mathrm{x}$ & & $\mathrm{x}$ & $\mathrm{x}$ & \\
\hline $\begin{array}{l}\text { Estabilidade entre a venda, previsão e } \\
\text { produção, reduzindo os estoques. }\end{array}$ & $\mathrm{x}$ & $\mathrm{x}$ & $\mathrm{x}$ & $\mathrm{x}$ & \\
\hline Redução de custos & $\mathrm{x}$ & $\mathrm{x}$ & & & $\mathrm{x}$ \\
\hline
\end{tabular}

Observou-se que os responsáveis operacionais de ambas as empresas entendem como vantagem o fornecedor gerenciar a demanda do cliente. Segundo o Gerente Geral da fornecedora de latas: "Entendo que qualquer processo colaborativo é calcado em parceria ganha-ganha, pois muitas vezes aparecem concorrentes e começa-se a barganha por preço. Esse processo chega para reduzirmos tarefas e burocracias e, assim, reduzirmos custos e perpetuarmos a parceria". A empresa cliente entendeu como vantagem desse processo apenas a redução dos custos.

A terceira análise, no Quadro 4, foi sobre as dificuldades ocorridas na implementação do CPFR. Com os dados das entrevistas, criou-se um quadro, em que a coluna da esquerda apresenta todas as causas levantadas segundo os entrevistados. Essa coluna foi dividida em duas áreas: área superior, com as dificuldades que coincidiram com a literatura pesquisada, e área inferior, com as dificuldades que não coincidiram com a literatura pesquisada. As causas de ambas as áreas foram organizadas em ordem decrescente em relação à quantidade de citações pelos entrevistados. Observa-se que mais da metade das dificuldades identificadas pelos entrevistados coincidiu com a literatura pesquisada.

\section{Quadro 4. Percepções sobre as dificuldades na implementação do CPFR}

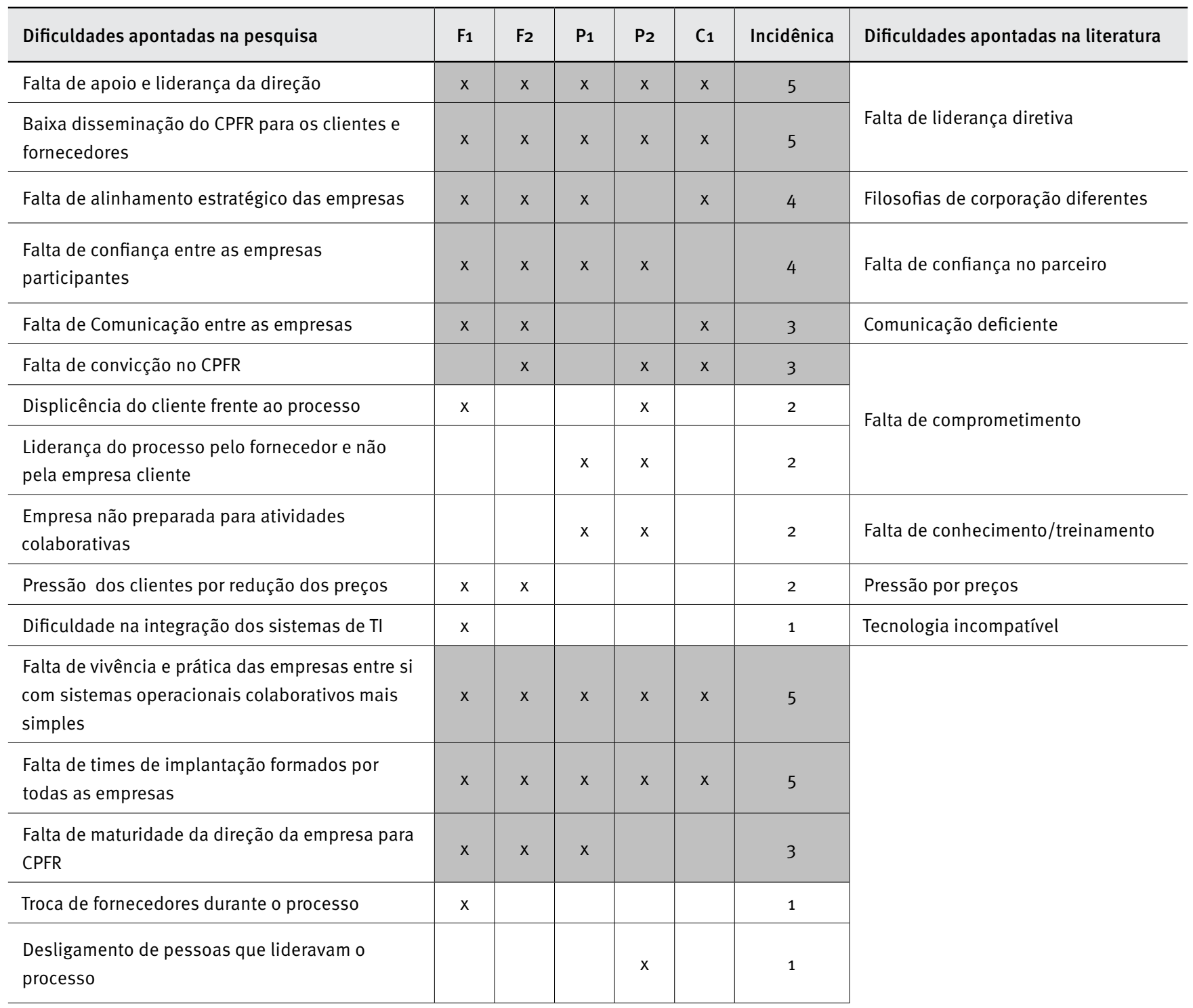




\section{Interpretação dos dados}

Perceberam-se, a partir da pesquisa, interdependências entre as dificuldades levantadas pelos entrevistados. Considerando essas interdependências, foi construído um mapa cognitivo no intuito de melhor visualizar as relações entre elas e identificar as causas raízes da implementação não efetiva do CPFR para o caso estudado. A Figura 2 mostra o mapa cognitivo.

Figura 2. Mapa cognitivo da realação dos fatores de fracasso na implementação do CPFR

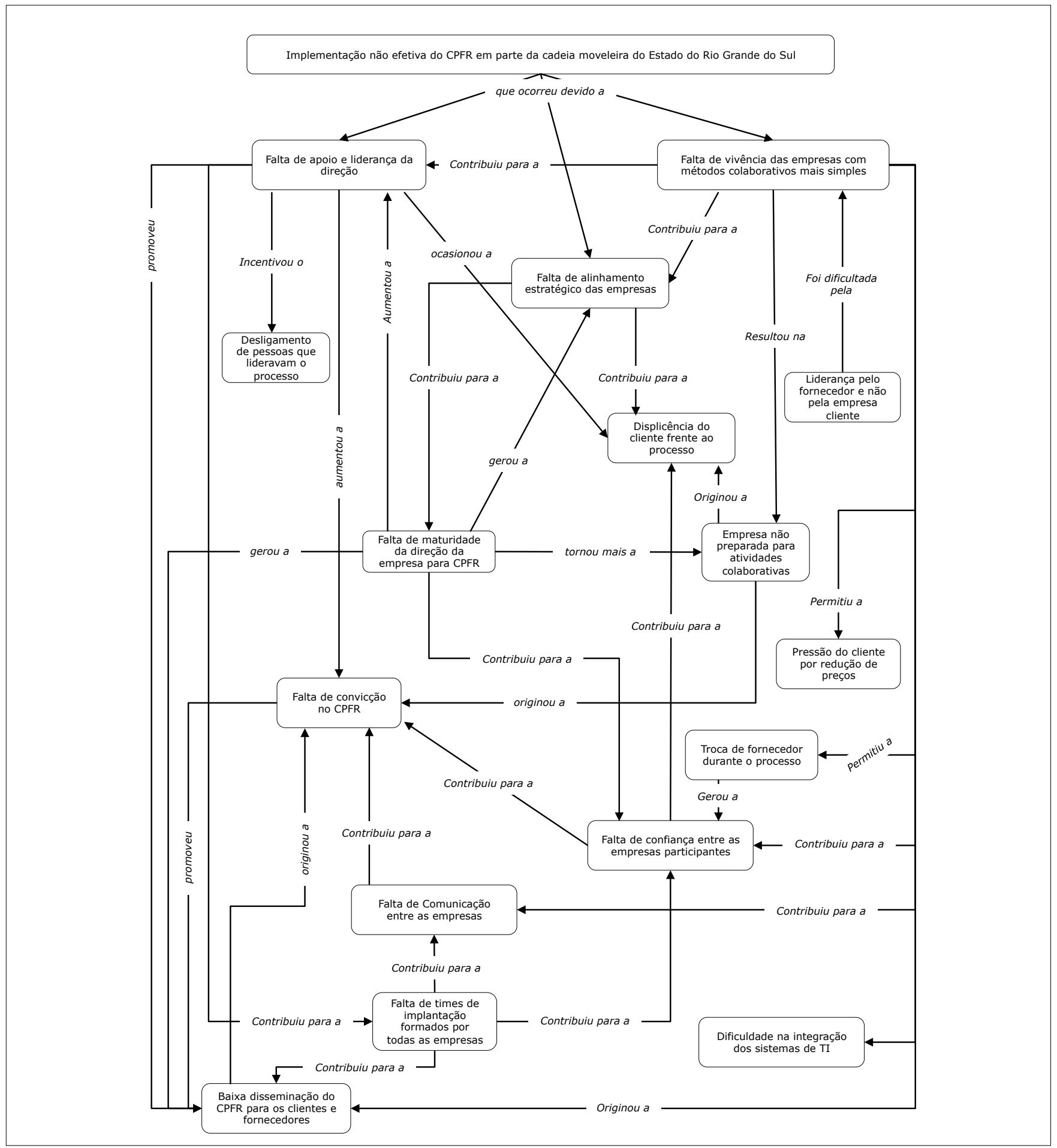


O mapa indica que duas dificuldades deram origem às demais: falta de apoio e liderança da direção; e falta de vivência das empresas com métodos colaborativos mais simples (como o VMI). Observa-se no mapa cognitivo que o maior número de entradas e saídas está associado a essas duas dificuldades, sugerindo a dependência de todo o processo em torno delas.

A primeira consta na literatura pesquisada como falta de liderança diretiva.

A segunda não é apontada na literatura, mas revela uma visão mais abrangente das dificuldades em implantações do CPFR. Refere-se à prática de relacionamento entre as empresas, contemplando a experiência com iniciativas colaborativas como preparação para implementações de métodos mais avançados como o CPFR. Essa ausência foi levantada no estudo de caso como a principal causa da implementação não efetiva do CPFR. Vivaldini, Pires, e Souza (2010), assim como outros autores mencionados anteriormente, afirmaram que cultura e confiança são fatores-chave para o sucesso na implementação de métodos colaborativos. Porém, esses autores não indicam como a cultura e confiança são desenvolvidas. Segundo os resultados do estudo de caso, esses fatores-chave podem ser desenvolvidos por meio da experiência com troca de informações e colaboração.

0 comprometimento da empresa distribuidora mostrou-se limitado, pois ela pouco colaborou, e só o fez devido uma oportunidade de novos negócios e redução de custos. Essa atitude caracterizou-se como um comportamento oportunista.

Apesar de o processo de implementação do CPFR não ter obtido o resultado almejado, observou-se a consequência positiva de servir para que as empresas aprimorassem seus relacionamentos e amadurecessem diante da GCS. Segundo conclusão do líder do projeto CPFR:

Conseguimos perceber que, primeiramente, as empresas devem ter suas bases de interesses estratégicos mútuos, pois, quando as empresas pertencem a cadeias diferentes, os interesses estratégicos tendem a ser diferentes e gerar conflitos. Então, a avaliação e alinhamento de interesses se fazem necessários [...] Posteriormente, as empresas devem iniciar as atividades colaborativas através de iniciativas simples, seja gerenciando o estoque do cliente, troca de tecnologias ou auxílio técnico. Assim, as empresas adquirem confiança, ajudando umas às outras a melhorar e ter sucesso. Após isso, as empresas podem então desenvolver métodos mais avançados como CPFR.

\section{CONCLUSÕES E RECOMENDAÇÕES PARA TRABALHOS FUTUROS}

O primeiro objetivo do artigo, que foi identificar os métodos colaborativos atualmente disseminados, apontou o CPFR como mais incidente, mais abrangente e mais completo em relação aos outros. Essa condição mostrou também que os métodos colaborativos para coordenação da CS ainda são pouco disseminados e praticados em relação a outras iniciativas colaborativas encontradas nos outros artigos não selecionados. A busca também poderia ser por meio do uso de outras palavras-chave, como iniciativa, ferramenta, prática, modelo e abordagem.

0 segundo objetivo do artigo, que foi levantar os potenciais fatores ou dificuldades na implementação de métodos colaborativos em CS, deu-se a partir da implementação não efetiva do CPFR do caso estudado. 0 resultado apresentou a falta de vivência das empresas com métodos colaborativos mais simples como a principal causa do insucesso no caso estudado e indicou que as empresas necessitam de uma preparação e experiências mínimas necessárias à implementação de métodos colaborativos mais avançados como o CPFR. Isso permitirá às empresas o aumento da confiança e do comprometimento ao longo do tempo, uma vez que percebem que o fato de se ajudarem traz melhorias. Esse fato fortalecerá a cultura colaborativa na CS.

A compreensão da CS está relacionada à interação entre as empresas, e a adoção de iniciativas colaborativas garantirá níveis de desempenho melhores e redução de custos. Ding, Guo, e Liu (2011) apontaram que, a fim de melhorar a competitividade da CS, sistemas de informação e métodos colaborativos são amplamente utilizados, e que a ênfase geralmente é dada à redução de custos por meio da otimização de processos, de inventário, de produção e de transporte. Porém, as típicas informações compartilhadas são níveis de inventários, planos de produção, previsão de demanda e capacidades de fornecimento. Corroborando essa ideia, a presente pesquisa demonstrou uma característica do CPFR voltada puramente à coordenação da CS, e não à otimização ou melhoria operacional. Logo, uma sugestão para trabalhos futuros é a busca por métodos colaborativos focados na otimização operacional da CS, que venha a complementar essa ausência identificada no CPFR ou em métodos similares.

\section{REFERÊNCIAS}

Andraski, J. (2001). CPFR: Collaborative Planning, Forecasting and Replenishment: A Necessity for Trading Partners. São Paulo: ECR.

Andraski, J. (2002). CPFR: Collaborative Planning, Forecasting and Replenishment. São Paulo: APAS.

Andraski, J. (2003). Getting CPFR: State of the supply chain, trends \& challenges. São Paulo: ECR Brasil.

Attaran, M., \& Attaran, S. (2007). Collaborative supply chain management: The most promising practice for building efficient and sustainable supply chains. Business Process Management Journal, 13(3), 390-404. doi:10.1108/14637150710752308 
Ayers, J. B. (2001). Introduction to the supply chain. In J. B. Ayers (Ed.). Handbook of supply chain management. Boca Raton, FL: St. Lucie Press.

Bailey, K., \& Francis, M. (2008). Managing information flows for improved value chain performance. International Journal of Production Economics, 111(1), 2-12. doi:10.1016/j.ijpe.2006.11.017

Baiman, S., \& Rajan, M. V. (2002). The role of information and opportunism in the choice of buyer-supplier relationships. Journal of Accounting Research, 40(2), 247-278. doi:10.1111/1475-679x.00046

Bandt, J. (1982). Les filieres de production: Mythe ou réalité. Economieet PME, (3)

Barratt, M., \& Oliveira, A. (2001). Exploring the experiences of collaborative planning initiatives. International Journal of Physical Distribution \& Logistics Management, 31(4), 266-289. doi:10.1108/09600030110394932

Begnis, H. S. M., Estivalete, V. F. B., \& Pedrozo, E. A. (2007). Confiança, comportamento oportunista e quebra de contratos na cadeia produtiva do fumo no sul do Brasil. Gestão \& Produção, 14(2), 311-322. doi:10.1590/s0104-530x2007000200009

Bellmunt, T. V., \& Torres, P. R. (2013). Integration: Attitudes, patterns and practices. Supply Chain Management: An International Journal, 18(3), 308-323. doi:10.1108/scm-04-2012-0116

Cannon, J. P., Doney, P. M., Mullen, M. R., \& Petersen, K. J. (2010). Building long-term orientation in buyer-supplier relationships: The moderating role of culture. Journal of Operations Management, 28(6), 506-521. doi:10.1016/j.jom.2010.02.002

Cao, M., \& Zhang, Q. (2011). Supply chain collaboration: Impact on collaborative advantage and firm performance. Journal of Operations Management, 29(3), 163-180. doi:10.1016/j.jom.2010.12.008

Carle, M., Martel, A., \& Zufferey, N. (2012). The CAT metaheuristic for the solution of multi-period activity-based supply chain network design problems. International Journal of Production Economics, 139(2), 664-677. doi:10.1016/j.ijpe.2012.06.016

Carr, A. S., Kaynak, H., \& Muthusamy, S. (2008). The cross-functional coordination between operations, marketing, purchasing and engineering and the impact on performance. International Journal of Manufacturing Technology and Management, 13(1), 55-77.

Cederlund, J. P., Kohli, R., Sherer, S. A., \& Yao, Y. (2007). How Motorola put CPFR into action? Supply Chain Management Review. Recupera do de http://www.scmr.com/

Chan, F. T. S., Chong, A. Y., \& Zhou, L. (2012). An empirical investigation of factors affecting e-collaboration diffusion in SMEs. International Journal of Production Economics, 138(2), 329-344. doi:10.1016/j. ijpe.2012.04.004

Chopra, S., \& Meindl, P. (2001). Supply chain management. Englewood Cliffs, NJ: Prentice-Hall.

Christopher, M. (1998). Logistics \& supply chain management: Strategies for reducing costs and improving services. London: Prentice-Hall.

Cuenca, L., Boza, A., Alemany, M. M. E., \& Trienekens, J. J. M. (2013). Structural elements of coordination mechanisms in collaborative planning processes and their assessment through maturity models: Application to a ceramic tile company. Computers in Industry, 64(8), 898-911. doi:10.1016/j.compind.2013.06.019

Danese, P. (2007). Designing CPFR collaborations: Insights from seven case studies. International Journal of Operations \& Production Management, 27(2), 181-204. doi:10.1108/01443570710720612
Ding, H., Guo, B., \& Liu, Z. (2011). Information sharing and profit allotment based on supply chain cooperation. International Journal of Production Economics, 133(1), 70-79. doi:10.1016/j.ijpe.2010.06.015

Doney, P. M., \& Cannon, J. P. (1997). An examination of the nature of trust in buyer-seller relationships. Journal of Marketing, 61(2), 35-51. doi:10.2307/1251829

Fabbe-Costes, N., \& Jahre, M. (2007). Supply chain integration improves performance: The Emperor's new suit?. International Journal of Physical Distribution \& Logistics Management, 37(10), 835-855. doi:10.1108/09600030710848941

Fisher, M. (2007). Strengthening the empirical base of operations management. Manufacturing \& Service Operations Management, 9(4), 368-382. doi:10.1287/msom.1070.0168

Fliedner, G. (2003). CPFR: An emerging supply chain tool.Industrial Management \& Data Systems, 103(1), 14-21.doi:10.1108/02635570310456850

Flynn, B. B., Huo, B., \& Zhao, X. (2010). The impact of supply chain integration on performance: A contingency and configuration approach. Journal of Operations Management, 28(1), 58-71. doi:10.1016/j. jom.2009.06.001

Frohlich, M. T., \& Westbrook, R. (2001). Arcs of integration: An international study of supply chain strategies. Journal of Operations Management, 19(2), 185-200. doi:10.1016/S0272-6963(00)00055-3

Ganesan, S. (1993). Negotiation strategies and the nature of channel relationships. Journal of Marketing Research, 30(2), 183-203. doi:10.2307/3172827

Gomes, L. C. (2014). Métodos colaborativos em cadeias de suprimentos. UPDATE-Revista de Gestão de Negócios, 1(1), 111-136.

Hill, C. W. (1990). Cooperation, opportunism, and the invisible hand: Implications for transaction cost theory. Academy of Management Review, 15(3), 500-513. doi:10.5465/AMR.1990.4309111

Hines, P. (1993). Integrated materials management: The value chain redefined. The International Journal of Logistics Management, 4(1), 13-22. doi:10.1108/09574099310804920

Holweg, M., \& Pil, F. K. (2008). Theoretical perspectives on the coordination of supply chains. Journal of Operations Management, 26(3), 389-406.

Ireland, R. (2005). Supply chain collaboration: How to implement CPFR and other best collaborative practices. Boca Raton: J. Ross Publishing Inc.

Kliemann, F. J., Neto, \& Souza, S. O. (2004). Desenho, análise e avaliação de cadeias produtivas. In Oliveira et al. (Eds.). Redes produtivas para o desenvolvimento regional. Ouro Preto: ABEPRO.

Koh, S. C. L., Demirbag, M., Bayraktar, E., Tatoglu, E., \& Zaim, S. (2007). The impact of supply chain management practices on performance of SMEs. Industrial Management \& Data Systems, 107(1), 103-124. doi:10.1108/02635570710719089

Kwon, I. W. G., \& Suh, T. (2004). Factors affecting the level of trust and commitment in supply chain relationships. Journal of Supply Chain Management, 40(1), 4-14. doi:10.1111/j.1745-493x.2004.tbo0165.x

Leuschner, R., Rogers, D. S., \& Charvet, F. F. (2013). A meta-analysis of supply chain integration and firm performance. Journal of Supply Chain Management, 4(2), 34-57. doi:10.1111/jscm.12013

Manthou, V., Vlachopoulou, M., \& Folinas, D. (2004). Virtual e-Chain (VeC) model for supply chain collaboration. International Journal of Production Economics, 87(3), 241-250. doi:10.1016/So9255273(03)00218-4 
Marks, C. (2005). Critical factors of CPFR: Collaborative, planning, forecasting \& replenishment. Proceedings of the Annual Napm-Nca Purchasing Bootcamp, Falls Church, 3.

Mattar, F. N. (1997). Pesquisa em marketing. São Paulo: Atlas.

Mentzer, J. (2001). Managing supply chain collaboration. In J. Mentzer (Ed). Supply chain management (pp. 83-84). Thousand Oaks: Sage Publications.

Min, S., Roath, A. S., Daugherty, P. J., Genchev, S. E., Chen, H., \& Arndt, A. D. (2005). Supply chain collaboration: What's happening?. The International Journal of Logistics Management, 16(2), 237-256. doi:10.1108/09574090510634539

Monczka, R. M., Petersen, K. J., Handfield, R. B., \& Ragatz, G. L. (1998). Success factors in strategic supplier alliances: The buying company perspective. Decision Sciences, 29(3), 553-577. doi:10.1111/j.1540-5915.1998.tbo1354.x

Nakano, M. (2009). Collaborative forecasting and planning in supply chains: The impact on performance in Japanese manufacturers. International Journal of Physical Distribution \& Logistics Management, 39(2), 84-105. doi :10.1108/09600030910942377

Narayanan, V. G., \& Raman, A. (2004). Aligning incentives in supply chains. Harvard Business Review. Recuperado de https://hbr.org/

Nyaga, G. N., Whipple, J. M., \& Lynch, D. F. (2010). Examining supply chain relationships: Do buyer and supplier perspectives on collaborative relationships differ? Journal of Operations Management, 28(2), 101-114. doi:10.1016/j.jom.2009.07.005

Porter, M. E. (1990). Vantagem competitiva. Rio de Janeiro: Campus.

Prahinski, C., \& Benton, W. C. (2004). Supplier evaluations: Communication strategies to improve supplier performance. Journal of Operations Management, 22(1), 39-62. doi:10.1016/j.jom.2003.12.005

Ramanathan, U. (2012). Supply chain collaboration for improved forecast accuracy of promotional sales. International Journal of Operations \& Production Management, 32(6), 676-695. doi:10.1108/01443571211230925

Ramanathan, U., \& Gunasekaran, A. (2012). Supply chain collaboration:
Impact of success in long-term partnerships. International Journal of Production Economics, 147(Part B), 252-259. doi:doi:10.1016/j. ijpe.2012.06.002

Sari, K. (2008). On the benefits of CPFR and VMI: A comparative simulation study. International Journal of Production Economics, 113(2), 575-586. doi:10.1016/j.ijpe.2007.10.021

Seifert, D. (2002). Collaborative planning forecasting and replenishment: How to create a supply chain advantage. Bonn: Galileo Business.

Sheffi, Y. (2002). The value of CPFR. Proceedings of RIRL Conference, Lisbon, Portugal.

Simchi-Levi, D., Kaminsky, P., \& Simchi-Levi, E. (2003). Designing and managing the supply chain: Concepts, strategies, and case studies (2nd ed.). New York: McGraw-Hill/Irwin.

Skjoett-Larsen, T., Thernøe, C., \& Andresen, C. (2003). Supply chain collaboration: Theoretical perspectives and empirical evidence. International Journal of Physical Distribution \& Logistics Management, 33(6), 531-549. doi:10.1108/09600030310492788

Smith, L. (2006). West marine: a CPFR success story. Supply Chain Management Review. Recuperado de http://www.scmr.com/.

Stadtler, H. (2009). A framework for collaborative planning and stateof-the-art. OR Spectrum, 31(1), 5-30. doi:10.1007/s00291-007-0104-5

Stank, T. P., Keller, S. B., \& Daugherty, P. J. (2001). Supply chain collaboration and logistical service performance. Journal of Business Logistics, 22(1), 29-48. doi:10.1002/j.2158-1592.2001.tbo0158.x

Voluntary Interindustry Commerce Stand. (2004). CPFR: An overview. New Jersey: VICS. Recuperado de http://committees.vics.org/committees/cpfr/CPFR_Overview_US-A4.pdf

Vivaldini, M., Pires, S. R. I., \& Souza, F. B. (2010). Importância dos fatores não-tecnológicos na implementação do CPFR. RAC-Revista de Administração Contemporânea, 14(2), 289-309. doi: 10.1590/S141565552010000200007

Williamson, O. E. (1985). The economic institutions of capitalism: Firms, markets, relational contracting. New York: The Free Press. 